\title{
DRIVER ASSESSMENT WITH MEASURES OF CONTINUOUS CONTROL BEHAVIOR
}

\author{
R. Wade Allen ${ }^{1}$, Thomas D. Marcotte ${ }^{2}$, Theodore J. Rosenthal ${ }^{1}$, Bimal L. Aponso ${ }^{1}$ \\ ${ }^{1}$ Systems Technology, Inc. \\ Hawthorne, California, USA \\ ${ }^{2}$ University of California at San Diego \\ San Diego, California, USA \\ E-mail: rwallen@,systemstech.com
}

\begin{abstract}
Summary: This paper reviews past research on stimulus/response analysis methods in continuous control tasks, and describes procedures for specifically measuring driver behavior in a car following task. Example driving simulator data is given for drivers with disease impairments. The data processing methods are summarized and example results are given to demonstrate the data analysis approach. Analysis of driver steering and speed control behavior have been used to identify normal highway operations and effects of various impairments, including drugs, alcohol, fatigue and medical conditions. Typical measures might include characteristics of control (steering, throttle, brake) activity, such as control reversals and expected values such as mean and standard deviation. More powerful time series analysis methods look at the relationship between stimulus and response variables. Fourier analysis procedures have been used to carry out stimulus/response relationships, such as steering response to wind gusts and roadway curvature, and speed response to lead vehicle speed variations. These methods allow the analysis of driver time delay in responding to stimulus inputs, and the correlation of driver response to the stimulus input. Typically, driver impairments lead to responses with increased time delay and decreased correlation.
\end{abstract}

\section{INTRODUCTION}

A variety of performance measures of driver and vehicle response can be employed for assessment. In field testing, measures of steering and speed control can be made along with vehicle motions including accelerations, yaw rate, and lane deviations. In simulations, additional measures might include time to collision and accidents given hazardous driving scenarios. In more benign scenarios, the measurement and characterization of continuous steering and speed control behavior is useful for driver assessment under conditions where the driver is impaired or the visual environment is degraded. A useful paradigm for assessing driver continuous control (e.g., steering, speed) involves stimulating the vehicle or driver in a controlled manner, then measuring the driver's response to the controlled stimulus. The stimulus can be applied as a wind gust, roadway curvature or grade, or with a lead vehicle with a controlled velocity profile. This paper gives a short review of continuous control assessment procedures, and describes the analysis of a simulated car following task by normal and impaired drivers.

\section{BACKGROUND}

A basic model for the human operator $(\mathrm{HO})$ performing a continuous control task is illustrated in Figure 1. Here the HO is attempting to control a vehicle (e.g., airplane, car) to respond to some 
stimulus (e.g., tracking another vehicle). These models and corresponding measurements have been well developed for the past several decades (McRuer, Allen, et al.,1977; Wierwille, Gagne and Knight, 1967). These methods allow the identification of the dynamic response of the HO (actions correlated with the stimulus) and noise or remnant processes that are uncorrelated with the stimulus but are an inherent component of the HO's response. The noise processes have been characterized as broad band and proportional to the activity in the error signal perceived by the HO, and also proportional to the HO's response (Jex and Magdaleno, 1969; Levison and Kleinman, 1968). Measures of continuous HO behavior have been obtained under normal and impaired conditions (Weir and McRuer, 1973; Allen, Jex, et al., 1975 ; Allen, O’Hanlon, et al., 1979).

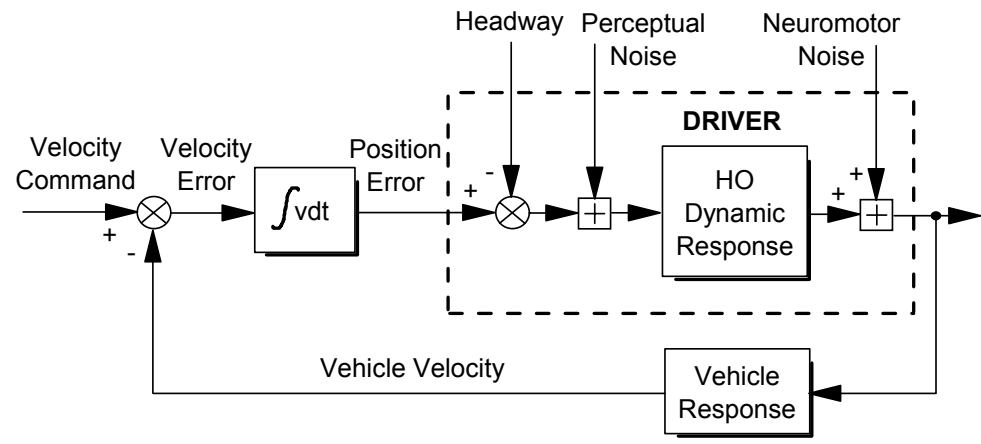

\section{Figure 1. Model of a Driver Performance a Car Following Task}

The HO modeling and analysis portrayed in Figure 1 has more recently been applied to driver longitudinal (speed) control (Allen, Magdaleno, et al., 1997). Instrumented vehicle data was collected under test track and open road conditions. In this database the authors identified HO gain and time delay basically identifying the band width or frequency response of the closed loop control process. This is important, because it tells us what kind of stimulus conditions would be reasonable to set up for identifying $\mathrm{HO}$ behavior in a speed control measurement paradigm. Figure 2 shows data on typical bandwidths measured in $\mathrm{HO}$ steering and speed control tasks.

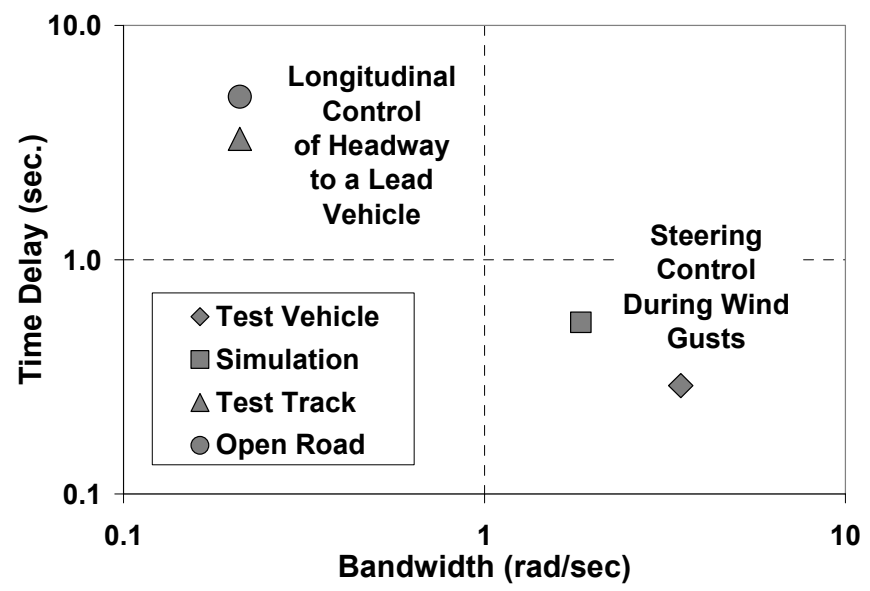

Figure 2. Bandwidths for HO Continuous Control (Allen, Jex, 1975; Allen, Magdaleno, et al., 1997) 
Here we see that the steering task has bandwidths on the order of ten times higher frequency than speed control, and that the bandwidths of speed control are down on the order of 0.25 radians/second (0.04 cycles/second or wave periods of 25 seconds). Stimulus measurement frequencies should primarily be near, and below, these bandwidth frequencies (e.g., wave lengths longer than 25 seconds) for the task to appear reasonable to subjects, and for us to get reasonable measures of driver time delays and remnant. Fourier analysis can then be used to process data and derive measures of time delay and remnant, as will be described below.

A general purpose Fourier measurement technique can be used to characterize the continuous control behavior (e.g., steering, throttle) of the human operator (e.g., driver, pilot, etc.). A sum of sine and cosine waves is defined for the task input, and the input and output of the human/machine system are measured and subjected to Fourier analysis. The Fourier coefficients can be computed for any signal in the control loop (e.g., lead vehicle stimulus or driver response) simply by mechanizing the finite sine and cosine transforms (e.g., Aseltine, 1958):

$$
\begin{aligned}
& F_{c}[x(t)]_{k}=\operatorname{Re} X\left(j \omega_{k}\right)=\frac{2}{T} \int_{t_{0}}^{t_{0}+T} x(t) \cos \left(\omega_{k} t\right) d t \\
& F_{s}[x(t)]_{k}=\operatorname{Im} X\left(j \omega_{k}\right)=\frac{2}{T} \int_{t_{0}}^{t_{0}+T} x(t) \sin \left(\omega_{k} t\right) d t
\end{aligned}
$$

The measurement frequencies $\omega_{k}$ must have an integer number of cycles per the run length $T: T$

$$
\frac{\omega_{k}}{2 \pi} T=n_{k} \quad(\text { an integer })
$$

The finite Fourier transform of $x(t)$ at the input frequencies $n_{k}$ is then given by:

$$
\begin{aligned}
F[x(t)]_{k} & =\frac{2}{T} \int_{t_{0}}^{t_{0}+T} x(t) e^{-j \omega_{k}} d t \\
& =\frac{2}{T} \int_{t_{0}}^{t_{0}+T} x(t)\left[\cos \omega_{k} t-j \sin \omega_{k} t\right] d t
\end{aligned}
$$

If drivers are responding to the stimulus, so that their response $x(t)$ contains a component at the measurement frequency $\omega_{k}$ then the Fourier integrals will integrate over time. These integrals can then be interpreted in terms of an open loop transfer function and the Figure 1 compensatory loop can be expressed in Laplace transform terms of a response amplitude, phase lag and related time delay:

$$
Y_{p} \bullet Y_{c}=\omega_{c} e^{-\tau_{e} s} / s
$$

In the above crossover model characterization of manual control system dynamic behavior (McRuer and Krendal, 1974), crossover frequency is a measure of the system (driver/vehicle) bandwidth, and the product of crossover frequency and time delay is a measure of system damping:

$$
\text { Crossover Frequency (bandwidth) }=\omega_{c}
$$$$
\text { Phase Margin (system damping) }=\phi_{m}=\pi / 2-\omega_{c}(\mathrm{rad} / \mathrm{sec}) \times \tau_{\mathrm{e}}(\mathrm{sec})
$$ 
This HO behavior at the stimulus frequencies $\omega_{k}$ is considered the correlated portion of the response. All other components of the response are considered to be the noise or remnant portrayed in Figure 1.

The research discussed in this paper concerns driver headway and speed control during car following. The speed control aspects of car following can relate generally to driver closed loop response to aerodynamic disturbances and roadway geometry. The guidance aspects of car following include perception of lead vehicle headway and relative velocity and closed loop control of following distance. Past research was motivated by a desire to understand the dynamics of driver headway control in traffic streams (e.g., Pipes, 1953), and as a guide for setting the control characteristics of Automatic Cruise Control (ACC) Systems (e.g. Sayers, Fancher, et al., 1995). Bekey, et al. (1977) provided a useful summary of past work and further analysis on the general problem of driver lead car following. These early control models assumed that during car following, drivers attempt to minimize velocity differences with a lead vehicle (i.e., a well-defined stimulus). Data collected by Chandler, et al. (1958) has shown that the driver can be characterized according to a closed loop bandwidth (gain or crossover frequency) of $0.37 \mathrm{rad} / \mathrm{sec}$ and a time delay of 1.5 seconds in responding to changes in lead vehicle velocity, which is consistent with the results illustrated in Figure 1. Torf and Duckstein (1966) also have collected data on driver detection times for several levels of lead car acceleration. They found detection times of 1.9 seconds for a lead car acceleration of $2.5 \mathrm{ft} / \mathrm{sec}^{2}$, and 2.5 seconds at an acceleration of $1.6 \mathrm{ft} / \mathrm{sec}^{2}$. By regression analysis they also determined that response time decreased by 0.8 seconds for each increase of $1 \mathrm{ft} / \mathrm{sec}^{2}$ in lead car acceleration. This may relate to the amount of time required to sense a velocity change (i.e., higher accelerations give more rapid change in lead car range and range rate).

\section{RESULTS}

A driving simulation experiment was performed on subjects having disease impairments. A description of a related experiment with multiple sclerosis (MS) patients and complete results are reported elsewhere at this conference (Marcotte, 2005). Data processing results will be reported here to illustrate the Fourier analysis procedures discussed previously, and to give some insight into the methodology and potential performance metrics. The experimental task was to follow a lead vehicle at a safe distance. The stimulus was applied to the lead vehicle's velocity. The mean velocity of the lead vehicle was $55 \mathrm{mph}$ (about $82 \mathrm{feet} / \mathrm{second}$ ), and the stimulus was a sinusoidal wave variation with an amplitude of six mph (about 9 feet/second) a period of one minute (60 seconds), or a frequency of 0.0167 cycles per second ( 0.095 radians/second). For simplicity, the experimental data were entered into a spreadsheet where the Fourier integrals, model computations and plotting were carried out.

Figure 3 shows speed stimulus and subject speed response time histories for several subjects along with Fourier integrals for the subject response (the Fourier integrals do not have the scale factor $2 / T$ ). Here we see three of the subjects following the velocity variations with some reasonably small error. Four of the subjects show various problems in following the stimulus. The subjects who follow the stimulus reasonably well also show well behaved Fourier integrals that identify their behavior. The waviness in the integrals occurs at twice the input frequency of the stimulus due to trigonometric identities, and the steady increase in the integrals shows that the subjects are 


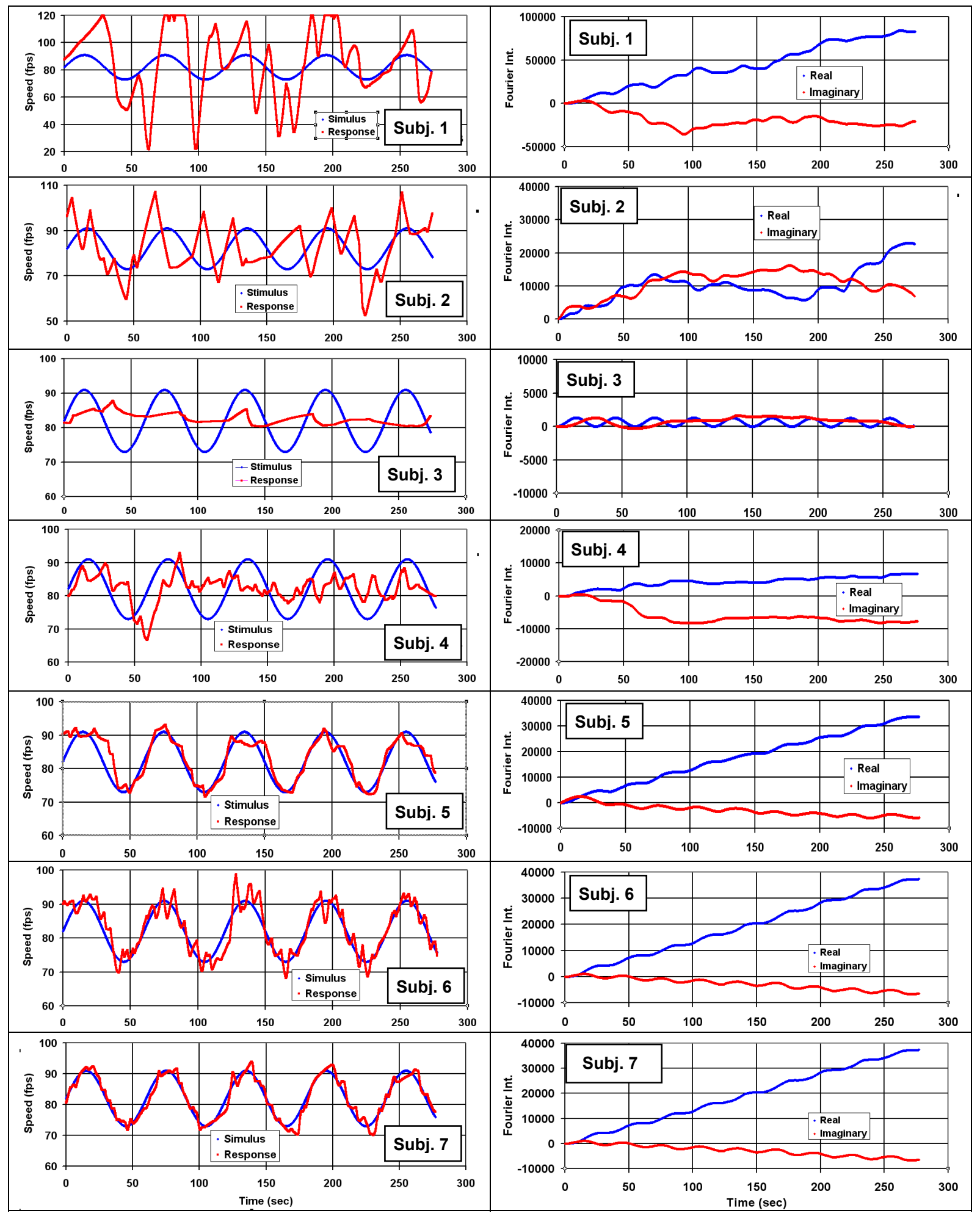

a) Time Histories

b) Fourier Integrals

Figure 3. Example Time Histories and Fourier Integrals for Lead Car Speed (Stimulus) and Subject Speed (Response) 
responding at the stimulus frequency. The four subjects demonstrating control response problems have Fourier integrals that are variable or minimal, and do not show consistent response behavior. Subject response analysis results based on the Fourier integrals illustrated in Figure 3 are summarized in Table 1. The ratio of subjects' responses at the stimulus frequencies to the total variance of the response is analogous to a correlation coefficient. Here we see that four of the subjects had very little correlation between their response and the stimulus which is somewhat obvious from their time histories and Fourier integrals in Figure 3. In these cases the amplitude ratios and time delays are unreliable. The three subjects with consistent control responses had high correlations, and the amplitude ratios and time delays provide reliable data. The amplitude ratios are close to unity, and the time delays are consistent with previous research on driver car following behavior.

Table 1. Subject Fourier Analysis Results Summary

\begin{tabular}{|c|c|c|c|c|}
\hline Subject & Amplitude Ratio & $\begin{array}{c}\text { Phase Lag } \\
\text { (radians) }\end{array}$ & $\begin{array}{c}\text { Time Delay } \\
\text { (seconds) }\end{array}$ & $\begin{array}{c}\text { Correlation } \\
\text { Coefficient }\end{array}$ \\
\hline 1 & 2.505 & 0.315 & 3.01 & 0.381 \\
\hline 2 & 0.591 & -0.513 & -4.89 & 0.133 \\
\hline 3 & 0.026 & 0.317 & 3.02 & 0.0127 \\
\hline 4 & 0.298 & 0.925 & 8.84 & 0.209 \\
\hline 5 & 0.964 & 0.196 & 1.87 & 0.874 \\
\hline 6 & 1.076 & 0.106 & 1.01 & 0.854 \\
\hline 7 & 1.067 & 0.184 & 1.76 & 0.965 \\
\hline
\end{tabular}

\section{DISCUSSION}

The correlation coefficient is probably the primary metric because very low values indicate that other metrics will be unreliable. Amplitude ratio also provides some measure of reliability. Amplitude ratios close to unity imply reasonable attention to the task. Low or high amplitude ratios correspond to low coherence behavior. Reliable time delays on the order of 1-2 seconds imply crossover frequencies with wave lengths on the order of 4-8 seconds. In the future, runs with shorter wave lengths than employed here could probably be used to make measurements procedures more efficient. Wave lengths on the order of 30-45 seconds might be used, with four waves giving run lengths on the order of 120-180 seconds (2-3 minutes). The Fourier analysis in general shows good stable measurements for subjects properly performing the car following task. The coherence measure provides a metric for subject consistency in performing the task. Finally, data analysis can easily be performed using spreadsheets. Fast Fourier Transform (FFT) methods can be used, but the data sampling frequency must be consistent with the FFT routine. FFT methods will also allow the full response spectrum to be analyzed, which then allows the driver's noise or remnant spectrum to be identified. 


\section{REFERENCES}

Allen, R.W. and Jex, H. R. (1972). A Simple Fourier Analysis Technique for Measuring the Dynamic Response of Manual Control Systems. IEEE Trans., Vol. SMC-2, No. 5, pp. 638-643. (STI-P-092)

Allen, R.W., Jex, H. R., et al. (1975). Alcohol Effects on Driving Behavior and Performance in a Car Simulator. IEEE Trans., Vol. SMC 5, No. 5, pp. 498-505.

Allen, R. W. and O'Hanlon, J. F. (1979). Effects of Roadway Delineation and Visibility Conditions on Driver Steering Performance. Transportation Research Record 739 Driver Performance, Passenger Safety Devices, and the Bicyclist, pp. 5-8. Washington, DC: Transportation Research Board.

Allen, R.W., Magdaleno, R.E., et al. (1997). Drvier Car Following Behavior Under Test Track and Open Road Driving Condition. SAE Paper 970170. Warrendale, PA: Society of Automotive Engineers.

Aseltine, J.A. (1958). Transform Method in Linear System Analysis. New York: McGraw-Hill.

Bekey, G.A., Burnham, G.O. and Seo, J. (1977). Control Theoretic Models of Human Drivers in Car Following. Human Factors, 19(4), pp. 399-413.

Chandler, F.E., Herman, R., and Montroll, E.W. (1958). Traffic Dynamics: Studies in Car Following. Operations Research, 6, pp. 165-184.

Jex, H.R., Magdaleno, R.E. (1969). Corroborative Data on Normalization of Human Operator Remnant. IEEE Transactions on Man-Machine Systems, MMS-10, No. 4, pp. 137-140.

Levison, W.H., Kleinman, D.L. (1968). A Model for Human Controller Remnant. Presented at the $19684^{\text {th }}$ Annual NASA-University Conference on Manual Control, University of Michigan, NASA SP 192.

Marcotte, T.D., Rosenthal, T.J., Corey-Bloom, J., Roberts, E., Lampinen, S., and Allen, R.W. (2005). The Impact of Cognitive Deficits and Spasticity on Driving Simulator Performance in Multiple Sclerosis. Presented at the 3rd International Driving Symposium on Human Factors in Driver Assessment, Training, and Vehicle Design, Rockport, Maine.

McRuer, D.T., Allen, R.W., et al. (1977). New Results in Driver Steering Control Models. Human Factors, 19(4), pp. 381-397.

McRuer, D.T. and Krendel, E.S. (1974). Mathematical Models of Human Pilot Behavior. AGARDogaph AGARD-AG-188.

McRuer, D.T. and Weir. D.H. (1969). Theory of Manual Vehicular Control. Ergonomics, 12(4), pp. 599-633.

Pipes, L.A. (1953). An Operational Analysis of Traffic Dynamics. Journal of Applied Physics, 24, pp. 271-281.

Sayers, J.R., Fancher, P.S., et al. (1995). Automatic Target Acquisition Autonomous Intelligent Cruise Control (AICC): Driver Comfort, Acceptance, and Performance in Highway Traffic. SAE Paper 950970. Warrendale, PA: Society of Automotive Engineers.

Torf, A.S. and Duckstein, L. (1966). A Methodology for the Determination of Driver Perceptual Latency in Car Following. Human Factors, 8(5), pp. 441-447.

Weir, D.H. and McRuer, D.T. (1973). Measurement and Interpretation of Driver/Vehicle System Dynamic Response. Human Factors, 15(4), pp. 367-378. 
PROCEEDINGS of the Third International Driving Symposium on Human Factors in Driver Assessment, Training and Vehicle Design

Wierwille, W.W., Gagne, G.A., and Knight, J.R. (1967). An Experimental Study of Human Operator Models and Closed-Loop Analysis Methods for High Speed Automobile driving. IEEE Transactions, HFE-8(3), 187-201. 